\title{
Vibronic fingerprint of singlet fission in hexacene
}

Cite as: J. Chem. Phys. 151, 054703 (2019); https://doi.org/10.1063/1.5110263

Submitted: 15 May 2019 . Accepted: 08 July 2019 . Published Online: 05 August 2019

Gang-Hua Deng, Qianshun Wei, Jian Han, Yuqin Qian, Jun Luo $\mathbb{D}^{\circ}$, Avetik R. Harutyunyan, Gugang Chen (D), Hongtao Bian (D), Hanning Chen (D), and Yi Rao (D)
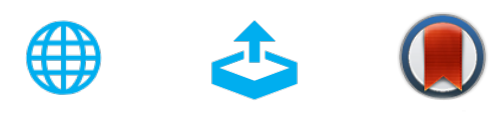

View Online

Export Citation

CrossMark

\section{ARTICLES YOU MAY BE INTERESTED IN}

Nonadiabatic simulation of singlet fission dynamics in tetracene clusters: The topology of quantum coherence in a global view

The Journal of Chemical Physics 151, 054308 (2019); https://doi.org/10.1063/1.5100196

Substituent effects on energetics and crystal morphology modulate singlet fission in 9,10bis(phenylethynyl)anthracenes

The Journal of Chemical Physics 151, 044501 (2019); https://doi.org/10.1063/1.5110411

Singlet fission in spiroconjugated dimers

The Journal of Chemical Physics 150, 204306 (2019); https://doi.org/10.1063/1.5097048

The Journal

Submit Today

of Chemical Physics

The Emerging Investigators Special Collection and Awards Recognizing the excellent work of early career researchers! 


\title{
Vibronic fingerprint of singlet fission in hexacene
}

\author{
Cite as: J. Chem. Phys. 151, 054703 (2019); doi: 10.1063/1.5110263 \\ Submitted: 15 May 2019 - Accepted: 8 July 2019 • \\ Published Online: 5 August 2019
}

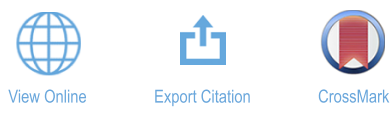

\author{
Gang-Hua Deng, ${ }^{1, a)}$ Qianshun Wei, ${ }^{2, a)}$ Jian Han, ${ }^{1,3, a)}$ Yuqin Qian, ${ }^{1}$ Jun Luo, ${ }^{3}$ (D) Avetik R. Harutyunyan, \\ Gugang Chen, (D) Hongtao Bian, ${ }^{2, b)}$ (D) Hanning Chen, ${ }^{5, b)}$ (D) and Yi Rao
}

\begin{abstract}
AFFILIATIONS
'Department of Chemistry and Biochemistry, Utah State University, Logan, Utah 84322, USA

${ }^{2}$ Key Laboratory of Applied Surface and Colloid Chemistry, Ministry of Education, School of Chemistry and Chemical Engineering, Shaanxi Normal University, Xi'an 710119, China

${ }^{3}$ School of Chemical Engineering, Nanjing University of Science and Technology, Nanjing 210094, China

${ }^{4}$ Honda Research Institute USA, Inc., San Jose, California 95134, USA

${ }^{5}$ Department of Chemistry, George Washington University, Washington, DC 20052, USA
\end{abstract}

Note: This paper is part of the JCP special collection on Singlet Fission.

a) Contributions: G.-H. Deng, Q. Wei, and J. Han contributed equally to this work.

${ }^{b)}$ Authors to whom correspondence should be addressed: htbian@snnu.edu.cn; chenhanning@gwu.edu; and yi.rao@usu.edu

\begin{abstract}
Singlet fission has the great potential to overcome the Shockley-Queisser thermodynamic limit and thus promotes solar power conversion efficiency. However, the current limited understandings of detailed singlet fission mechanisms hinder a further improved design of versatile singlet fission materials. In the present study, we combined ultrafast transient infrared spectroscopy with ab initio calculations to elucidate the roles played by the vibrational normal modes in the process of singlet fission for hexacene. Our transient infrared experiments revealed three groups of vibrational modes that are prominent in vibronic coupling upon photoexcitation. Through our computational study, those normal modes with notable Franck-Condon shifts have been classified as ring-twisting modes near $1300.0 \mathrm{~cm}^{-1}$, ring-stretching modes near $1600.0 \mathrm{~cm}^{-1}$, and ring-scissoring modes near $1700.0 \mathrm{~cm}^{-1}$. Experimentally, a ring-stretching mode near $1620.0 \mathrm{~cm}^{-1}$ exhibits a significant blue-shift of $4.0 \mathrm{~cm}^{-1}$ during singlet fission, which reaction rate turns out to be $0.59 \pm 0.07 \mathrm{ps}$. More interestingly, the blue-shifted mode was also identified by our functional mode singlet fission theory as the primary driving mode for singlet fission, suggesting the importance of vibronic coupling when a correlated triplet pair of hexacene is directly converted from its first excited state singlet exciton. Our findings indicate that the ultrafast transient infrared spectroscopy, in conjunction with the nonadiabatic transition theory, is a powerful tool to probe the vibronic fingerprint of singlet fission.
\end{abstract}

Published under license by AIP Publishing. https://doi.org/10.1063/1.5110263

\section{INTRODUCTION}

Singlet fission (SF) is a spin-allowed conversion process, in which a singlet exciton $\left(\mathrm{S}_{1} \mathrm{~S}_{0}\right)$ generated by a high energy photon splits into two correlated triplet excitons (TT). ${ }^{1,2}$ SF has received a great deal of attention since this process has the great potential to overcome the Shockley-Queisser limit and thus achieves unprecedented solar power conversion efficiencies. ${ }^{2,3}$ Many experimental $^{4-25}$ and theoretical ${ }^{26-45}$ efforts have been devoted to improve our understandings of this critical photochemical process, particularly at the molecular level, when designing rational photovoltaic systems. Nevertheless, the detailed SF mechanisms are still under hot debate due to the complicated convolutions of spin dynamics and electronic and vibrational relaxation, in particular, the interactions between electronic and vibrational states, namely, vibronic coupling. ${ }^{31}$ Therefore, it is imperative to develop a powerful ultrafast spectroscopic tool to probe the subtle yet critical vibronic fingerprint of SF that may occur on subpicosecond time scales.

Owing to the recent advances in ultrafast spectroscopies, the vibrational normal modes that are essential to SF can be uniquely identified through the time-resolved transient pump-probe technique. ${ }^{9,19-21}$ For instance, in a vibronic spectroscopy experiment, a normal mode of $1567 \mathrm{~cm}^{-1}$ exhibits the strongest spontaneous Raman signal arising from the conical intersection between $S_{1} S_{0}$ 
and TT states. ${ }^{20}$ In our previous theoretical study, SF was found to be facilitated by a few aromatic-ring-stretching modes centered at $1580 \mathrm{~cm}^{-1}$ in single-crystalline pentacene. ${ }^{46}$ According to the nonadiabatic transition theory, ${ }^{47}$ in the absence of light irradiation, energy conservation between reactant and product electronic states can be fulfilled only through thermal fluctuations, which also drive the system toward the diabolic point where the nonadiabatic coupling is nonvanishing. In organic molecular crystals such as tetracene, pentacene, and hexacene, their normal modes can be readily characterized, thanks to crystalline symmetry and weak interactions between lattice-site occupants. As an example, our measured steady-state Fourier transform infrared (FTIR) spectrum for a hexacene crystal only exhibits a few prominent peaks [Fig. 1(a)], suggesting the necessity of transient IR spectroscopy on monitoring vibrational wave packets during SF in organic crystals. In fact, ultrafast transient IR spectroscopy has been utilized by Asbury $^{48,49}$ and Wasielewski ${ }^{50,51}$ groups to track the formation of correlated triplet pairs through time-dependent mid-infrared transitions. ${ }^{48}$ Their studies on covalent terrylenediimide dimers indicated that the multiexciton intermediate possesses mixed characteristics of $S_{1} S_{0}$, charge transfer (CT), and TT states. ${ }^{50}$ Nevertheless, to the best of our knowledge, no time-resolved transient IR experiment has been conducted to uniquely ascertain SF-driving normal modes that could be justified by our functional mode singlet fission (FMSF) theory. To this end, our proposed combination of transient IR spectroscopy and FMSF theory in the present study will offer a complimentary tool set for investigating vibronic coupling in SF for a more efficient light-to-electricity conversion.
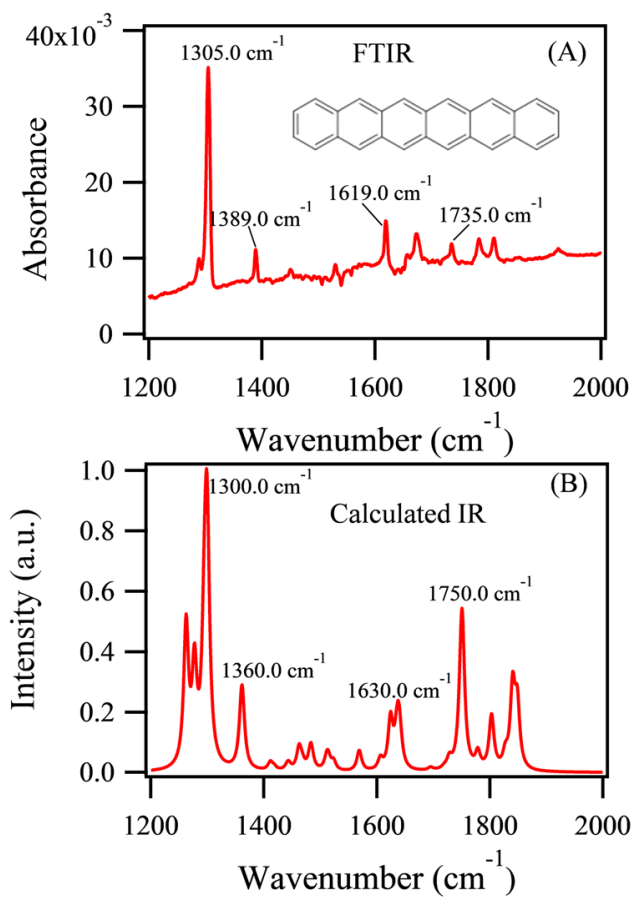

FIG. 1. (a) Steady-state FTIR of the hexacene thin film grown on the $\mathrm{CaF}_{2}$ window. (b) Calculated normalized infrared intensity for ground state hexacene.

\section{EXPERIMENTAL DETAILS}

Our experimental setup for a visible pump and a mid-IR probe measurement is similar to the ultrafast IR spectroscopy measurement for liquid samples, which has been described in previous reports. ${ }^{52,53}$ Briefly, a femtosecond amplifier (SPITFIRE ACE$35 \mathrm{~F} 1 \mathrm{~K}$, Spectra Physics) with an output wavelength of $801 \mathrm{~nm}$ and a pulse duration of $40 \mathrm{fs}$ was seeded with the ultrafast short pulse from a Ti-sapphire oscillator (Element 20-1200, Newport Corporation). The femtosecond amplifier pumped an optical parametric amplifier (OPA, TOPAS, Light conversion) to produce a broadband mid-IR pulse with the following specifications: $\sim 140$ fs pulse duration, tunable frequency range from 3 to $15 \mu \mathrm{m}, \sim 200 \mathrm{~cm}^{-1}$ bandwidth, and $1 \mathrm{kHz}$ repetition rate. In the visible pump-IR probe experiments, the $801 \mathrm{~nm}$ output from the femtosecond amplifier was used as the excitation beam. Its excitation fluence was controlled by a neutral density filter (NDF) and was kept low to avoid exciton-exciton annihilation in hexacene. The femtosecond IR pulse was used as the probe beam where the frequency was resolved by a spectrograph and was detected by a liquid nitrogen-cooled $\mathrm{HgCdTe}$ (MCT) array detector (FPAS-6414, Infrared Systems Development). The IR signal was monitored by recording the transient absorption change of the IR probe beam. Two polarizers were placed to selectively measure the parallel or perpendicular polarized signal relative to the excitation beam. The time delay between the excitation and probe beams was controlled by a mechanical delay stage. The data acquisition at different time delays was automated by a computer program. The ultrafast spectroscopic measurements were carried out at room temperature $\left(22 \pm 1^{\circ} \mathrm{C}\right)$, and the humidity in our laboratory was controlled at $\sim 40 \%$.

Hexacene precursors were synthesized by following a previously reported protocol $^{54}$ before hexacene films were fabricated through chemical vapor deposition using $\mathrm{CaF}_{2}$ as the substrate. Specifically, the $\mathrm{CaF}_{2}$ substrate was first cleaned by piranha solution before being rinsed with de-ionized water. Afterwards, it dried up in vacuum. Finally, hexacene films were deposited onto the $\mathrm{CaF}_{2}$ substrate at a rate of $0.5 \AA / s$ in a vacuum of $6 \times 10^{-6}$ Torr. A typical film thickness was kept at $\sim 100 \mathrm{~nm}$, which was confirmed by UV-Vis absorption.

\section{COMPUTATIONAL DETAILS}

The single-crystalline hexacene was represented in our computational study as a $9 \times 9 \times 3$ supercell $^{54}$ with a total of 20412 atoms, as shown in Scheme 1. To achieve a compromised balance between numerical efficiency and physical accuracy, the hybrid quantum mechanics/molecular mechanics (QM/MM) approach ${ }^{55}$ was adopted. More specifically, the QM subsystem consists of a hexacene tetramer and its nearest-neighboring molecules that span along crystal axes $a$ and $b$ to form the reaction center of singlet fission, while all other hexacene molecules embody the MM subsystem. The QM subsystem was modeled by Goedecker-Teter-Hutter (GTH) pseudopotential, ${ }^{56}$ optimally tuned range-separated hybrid Perdew-Burke-Ernzerhof ( $\omega$-RSH-PBE) exchange-correlation functional, ${ }^{57}$ polarized-valence triple- $\zeta$ (PVTZ) basis set, ${ }^{58}$ electrostatic QM/MM coupling scheme, ${ }^{59}$ and wavelet-based Poisson solver. ${ }^{60}$ By contrast, the thermal fluctuation of the MM subsystem was described by the generalized AMBER empirical force field (GAFF). ${ }^{61}$ 
SCHEME 1. Molecular structure of a $9 \times 9 \times 3$ supercell of single-crystalline hexacene. The designated reaction center of singlet fission is highlighted. The molecular pair along the long crystal axis is colored blue, while its counterpart along the short crystal axis is colored red.

Unless otherwise specified, all QM/MM simulations were performed using the $\mathrm{CP} 2 \mathrm{~K}$ package.

\section{RESULTS AND DISCUSSION}

\section{A. Steady-state vibrational fingerprint of hexacene}

Figure 1(a) depicts FTIR spectra of a hexacene crystalline film on a $\mathrm{CaF}_{2}$ window. A prominent peak is located at $1305.0 \mathrm{~cm}^{-1}$ besides several weak peaks around 1389.0, 1620.0, and $1735.0 \mathrm{~cm}^{-1}$. The normal modes of the designated reaction center, which is a hexacene tetramer (Scheme 1), were first ascertained by diagonalizing its mass-weighted Hessian matrix after geometry optimization. Then, under a double harmonic approximation, ${ }^{63}$ its infrared spectrum was calculated by the following equation:

$$
I_{i}=\frac{N \pi}{3 c^{2}}\left|\frac{\partial \mu}{\partial Q_{i}}\right|^{2}
$$

where $I_{i}$ is the infrared intensity of the $i$ th normal mode, $Q_{i}, N$ is Avogadro's number, $c$ is the speed of light, and $\mu$ is the electric dipole moment. As shown in Fig. 1(b), the most prominent peak of our calculated IR spectrum appears at $1300.0 \mathrm{~cm}^{-1}$, in excellent agreement with its experimental counterpart at $1304.0 \mathrm{~cm}^{-1}$. Therefore, the main peak in our experimental FTIR spectrum at $1304.0 \mathrm{~cm}^{-1}$ was assigned to ring twisting, while the two other peaks at $1619.0 \mathrm{~cm}^{-1}$ and $1735.0 \mathrm{~cm}^{-1}$ were assigned to ring-stretching and ring-scissoring modes, respectively.

\section{B. Excited-state vibrational fingerprint of hexacene}

Figure 2(a) shows pseudocolor plots of ultrafast transient IR absorption spectra of the hexacene $(\Delta T / T)$ thin film from $1280.0 \mathrm{~cm}^{-1}$ to $1820.0 \mathrm{~cm}^{-1}$. The transient IR absorption spectra

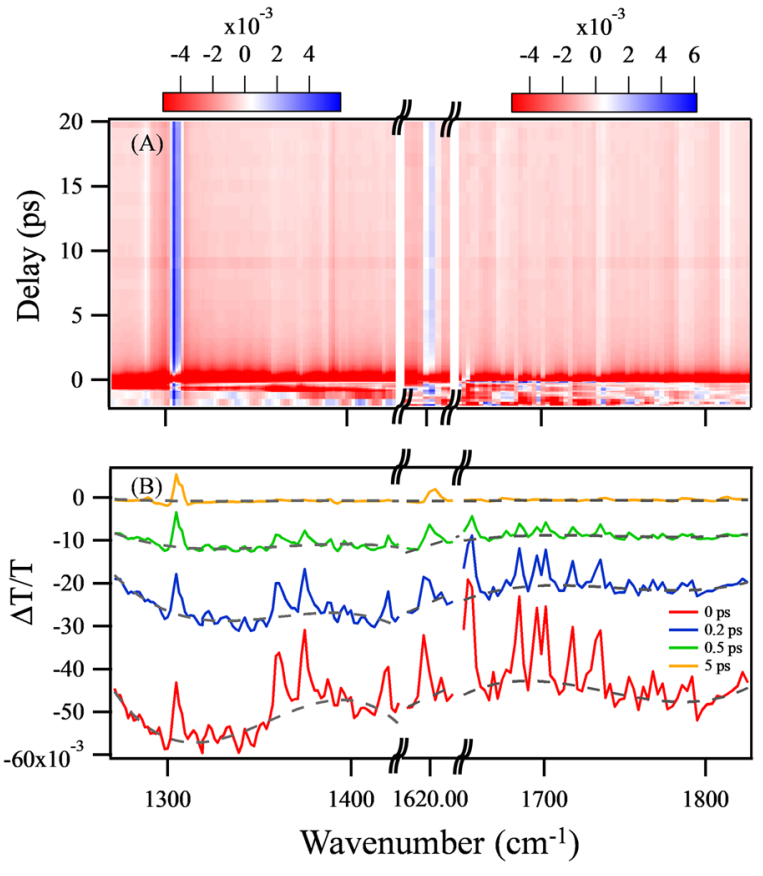

FIG. 2. (a) Pseudocolor plots of transient IR absorption spectra from the hexacene thin film excited at $801 \mathrm{~nm}$. (b) Ultrafast transient IR absorption spectra at the selected time delays. The dotted curves are the broad photoinduced absorption (PIA) from polaron.

consist of a broad photoinduced absorption (PIA) plus and a few narrow vibrational peaks. Figure 2 (b) also presents transient spectra at several time delays. The transient IR absorption can be readily decomposed into a broad and negative envelope in addition to several narrow positive peaks as presented at a time delay of $0 \mathrm{ps}$ in Fig. S1. It was found that the transient IR spectra are significantly different than those at the ground state. Previous studies of TIPS-pentacene have claimed that the prompt broad PIA is due to the absorption of singlet excited states and correlated triplet pair intermediates. ${ }^{48}$ Moreover, this broad feature was also assigned to the mid-IR absorption of polarons, ${ }^{64-70}$ suggesting the formation of ephemeral polarons in the irradiated hexacene thin film. This polaron generation occurs almost immediately upon photoexcitation [Fig. 2(a)] as was reported before. ${ }^{71,72}$ Specifically, Guo and co-worker observed polaron pairs on a time scale of $<100 \mathrm{fs}$ in RRa-P3HT and RR-P3HT films, ${ }^{71}$ while Lienau and co-workers reported a sub-20-fs time scale in a prototypical polymer thin film.? As will be discussed later, the polaron recombination also takes place on the order of a few hundred femtoseconds due to the small dielectric constant of hexacene. To assign the photoinduced narrow peaks, we categorized all of them into long-lived and short-lived ones. In general, a positive signature in change of transmission corresponds to ground state bleaching (GSB) or stimulated emission (SE), whereas a negative signature denotes photoinduced absorption. A majority of the peaks are short-lived except the one at $1304 \mathrm{~cm}^{-1}$, which is long-lived and eventually becomes positive. We attributed the relaxation process at $1304 \mathrm{~cm}^{-1}$ to the ground state recovery of hexacene. 
To further reveal the nature of the short-lived narrow peaks, we turn to theoretical calculations of the normalized infrared absorption intensity upon the $S_{0} S_{0} \rightarrow S_{1} S_{0}$ photoexcitation of hexacene. Specifically, the intensity was determined from the derivative of optical gap with respect to ground state geometry displacement. As shown in Fig. 3, the calculated intensity peaks are qualitatively consistent with those observed in Fig. 2. Moreover, the modes prominent in the infrared absorption intensity profile reflect three types of molecular vibrations: (a) ring-twisting modes $\left(1251.7 \mathrm{~cm}^{-1}\right.$, $1252.8 \mathrm{~cm}^{-1}, 1253.2 \mathrm{~cm}^{-1}, 1390.3 \mathrm{~cm}^{-1}$ ); (b) ring-stretching modes $\left(1597.1 \mathrm{~cm}^{-1}, 1597.7 \mathrm{~cm}^{-1}, 1598.7 \mathrm{~cm}^{-1}\right)$; and (c) ring-scissoring modes $\left(1757.1 \mathrm{~cm}^{-1}, 1757.7 \mathrm{~cm}^{-1}, 1779.6 \mathrm{~cm}^{-1}\right)$, as displayed in Fig. S6.

\section{Vibrational dynamics of hexacene}

We analyzed the dynamical behaviors of all vibrational modes that are important to vibronic coupling. The dynamics of the ringstretching mode near $1620.0 \mathrm{~cm}^{-1}$ at several time delays is shown in Fig. 4(a). Although it appears with a negative feature in the change of transmission, the peak overlaps with the broad featureless polaron background. In fact, the peak is positive in sign after subtracting the negative background. We attributed the peak near $1620.0 \mathrm{~cm}^{-1}$ to vibrational SE upon photoexcitation. A single exponential function fitting yielded a vibrational relaxation time of $84.7 \pm 4.7$ ps. Inspection of the transient spectra at $1620.0 \mathrm{~cm}^{-1}$ reveals a peak shift from $1618.0 \mathrm{~cm}^{-1}$ to $1622.0 \mathrm{~cm}^{-1}$ as the delay changes from 0 ps to 3 ps [Fig. 4(b)]. This spectral shift was not expected if this peak is due to the ground state recovery. Therefore, we attributed it to the $\mathrm{S}_{1} \mathrm{~S}_{0} \rightarrow$ TT transition of SF. A single exponential function fitting of Fig. 4(b) yielded a SF time constant of $0.59 \pm 0.07 \mathrm{ps}$, which is well in line with the previous result of Busby et al. ${ }^{8}$ Although the blue shift of the $1618 \mathrm{~cm}^{-1}$ peak might partially arise from internal vibrational energy redistribution (IVR), its effect should not be predominant in hexacene because the triplet quantum yield of hexacene is nearly $200 \%$ through SF. ${ }^{8}$ Furthermore, the linewidth of this peak was broadened from 1.4 to $2.7 \mathrm{~cm}^{-1}$ as the delay changed from 0 ps to $3 \mathrm{ps}$, indicating rapid vibrational dephasing during SF.

On the other hand, the modes at $1304.7 \mathrm{~cm}^{-1}$ and $1389.0 \mathrm{~cm}^{-1}$ in Fig. 5(a) exhibit different dynamical behaviors in a long time scale

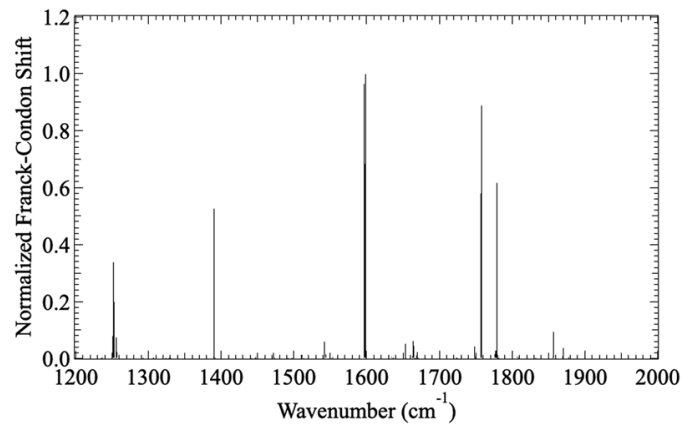

FIG. 3. Calculated normalized infrared absorption intensity upon the $S_{0} S_{0} \rightarrow S_{1} S_{0}$ photoexcitation in hexacene from the derivative of the optical energy gap with respect to the ground state geometry displacement.
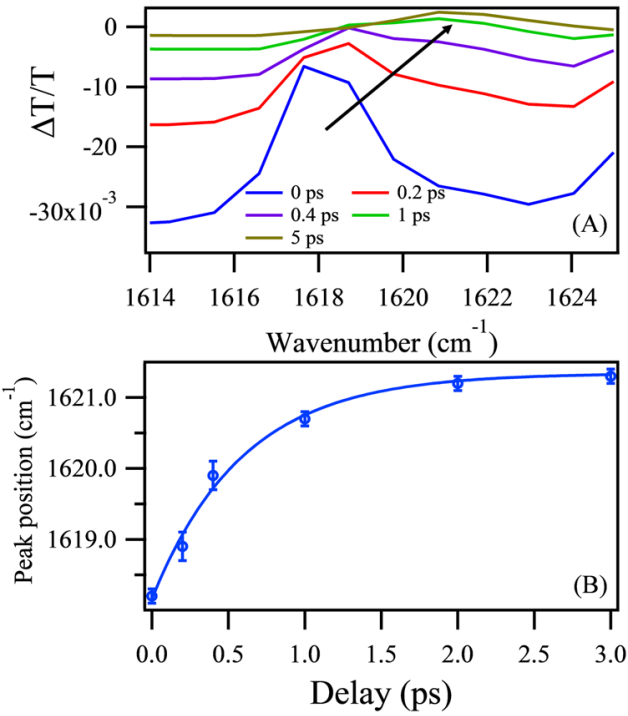

FIG. 4. (a) Ultrafast transient IR absorption spectra for the ring stretching mode near $1620.0 \mathrm{~cm}^{-1}$ at selected time delays. (b) Plot of the peak position as a function of the corresponding time delay. A single exponential fitting of the plot yielded a time constant of $0.59 \pm 0.07 \mathrm{ps}$.

of 1250 ps, despite their similar profiles in an early time window of 3 ps. The early kinetics with a negative signature arises from the abovementioned contribution of photoinduced polarons. The vibrational mode at $1304.7 \mathrm{~cm}^{-1}$ corresponds to the GSB peak of the in-plane $\mathrm{H}$ waging, exhibiting a slow relaxation process. The mode at $1389.0 \mathrm{~cm}^{-1}$ shows a fast recovery of $\sim 200 \mathrm{fs}$, and its peak manifests a slight shift with the time delay in Fig. S2. Kinetic traces at $1733.0 \mathrm{~cm}^{-1}$ and $1734.9 \mathrm{~cm}^{-1}$ for the ring-scissoring modes are presented in Figs. 5(c) and 5(d). These two peaks display a positive sign in the change of transmission, even though there are small contributions from the polaron at the early time window of a few hundred femtoseconds. The lifetime of these two peaks is on the order of a few hundred femtoseconds, excluding their origin from GSB. Therefore, they were assigned to the vibrational SE of hexacene. A single exponential function was used to fit the decay of the peaks at $1733.0 \mathrm{~cm}^{-1}$ and $1734.9 \mathrm{~cm}^{-1}$, yielding relaxation time constants of $193 \pm 10 \mathrm{fs}$ and $317 \pm 9 \mathrm{fs}$, respectively. The lifetimes of these two peaks for ring-scissoring modes are much shorter than the typical lifetime of a vibrational excited state. In addition, they afforded a slight spectral shift with the time delay in Fig. S3. Note that the rising time of the $1733.0 \mathrm{~cm}^{-1}$ mode occurs $\sim 100$ fs earlier than that of $1734.9 \mathrm{~cm}^{-1}$ [Fig. 5(b)]. This time difference was not due to spectral chirp because these two peaks are only separated by $2 \mathrm{~cm}^{-1}$. According to our proposed direct $S_{1} S_{0} \rightarrow T_{1} T_{1}$ transition, we assigned the peaks at $1733 \mathrm{~cm}^{-1}$ and $1735 \mathrm{~cm}^{-1}$ to the $S_{1} S_{0}$ and TT states, respectively. Therefore, the time evolution of these two peaks explicitly revealed the SF process of $S_{1} S_{0} \rightarrow T_{1} T_{1}$. Although it is still rather challenging to directly observe the optically dark TT state from spectroscopic data, we have shown that its time evolution can be monitored by transient IR. Nevertheless, the coupling of these $1730 \mathrm{~cm}^{-1}$ modes 


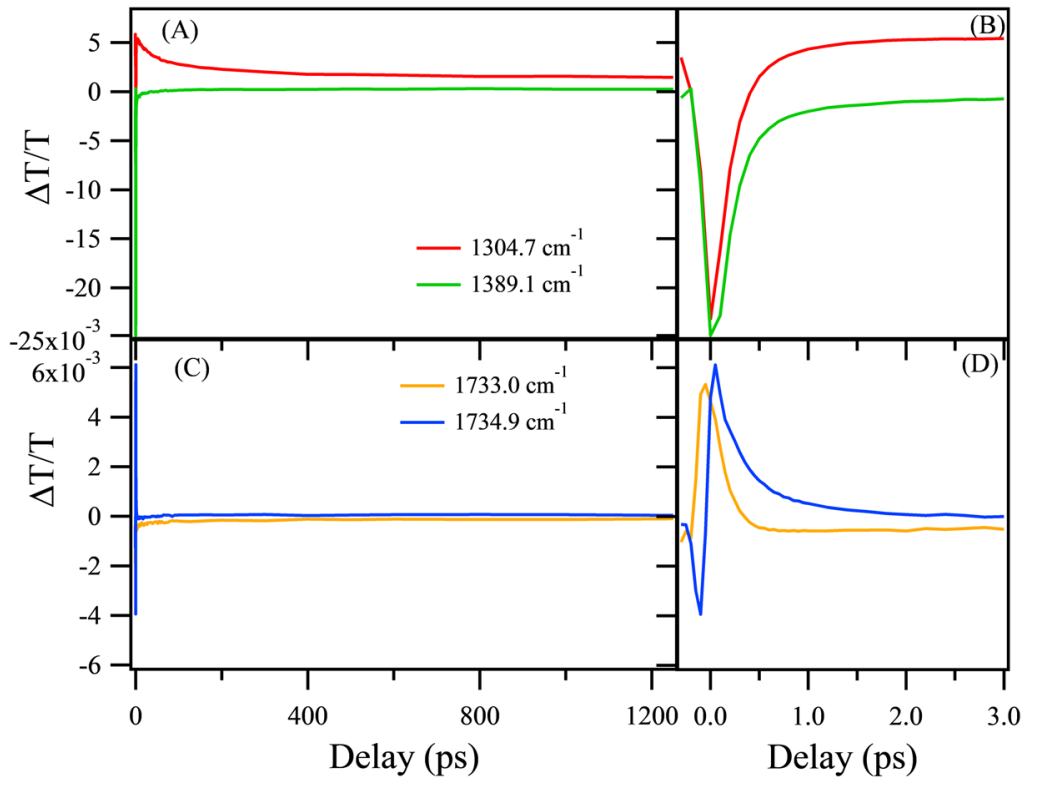

FIG. 5. [(a) and (b)] Kinetic traces at $1304.7 \mathrm{~cm}^{-1}$ and $1389.0 \mathrm{~cm}^{-1}$ from delay time -1 ps-1250 ps (a) and from delay time -1 ps-3 ps. [(c) and (d)] Kinetic traces at $1733.0 \mathrm{~cm}^{-1}$ and $1734.9 \mathrm{~cm}^{-1}$ from delay time $-1 \mathrm{ps}-1250 \mathrm{ps}$ and from delay time $-1 \mathrm{ps}-3 \mathrm{ps}$. with the $S_{1} S_{0} \rightarrow T_{1} T_{1}$ transition is much weaker than that of the $1620 \mathrm{~cm}^{-1}$ modes, which drive the formation of the TT state from the photoexcited singlet exciton $\mathrm{S}_{1} \mathrm{~S}_{0}$.

\section{Functional mode analysis for reaction coordinate of singlet fission}

In order to reveal the importance of vibronic coupling to SF, we performed functional mode analysis to identify its reaction coordinate. In condensed phase, an electron transfer or spin exchange process is typically driven by a collective motion of multiple normal modes. According to our functional mode electron transfer theory, ${ }^{74}$ the relative importance of a normal mode to a given nonadiabatic transition can be determined by linear least squares regression. More specifically, the reaction coordinate, $\vec{V}_{R C}$, is considered as a linear combination of all normal modes, $\vec{V}_{R C}=\sum_{i} c_{i} \vec{V}_{i}$. Then, the coefficient set, $\left\{c_{i}\right\}$, is optimized by maximizing Pearson's correlation coefficient, $R$, between the diabatic energy gap, $\Delta E_{d}$, and atomic displacement, $\vec{V}$,

$$
R=\frac{\operatorname{cov}\left(p_{\vec{V}_{R C}}(t), \Delta E_{d}(t)\right)}{\sigma_{\vec{V}_{R C}} \sigma_{\Delta E_{d}}},
$$

where $p_{\vec{V}_{R C}}(t)$ is the projection of instantaneous nuclear configuration on $\vec{V}_{R C}, \Delta E_{d}(t)$ is the instantaneous diabatic energy gap, $\operatorname{cov}\left(p_{\vec{V}_{R C}}(t), \Delta E_{d}(t)\right)$ is their covariance function, and their standard deviations are denoted as $\sigma_{\vec{V}_{R C}}$ and $\sigma_{\Delta E_{d}}$, respectively. Mathematically, the optimization of $R$ is achieved by solving the following coupled linear equations:

$$
\sum_{i=1}^{N_{v i b}} c_{i} \operatorname{cov}\left(p_{\vec{V}_{i}(t)}, p_{\vec{V}_{j}(t)}\right)=\operatorname{cov}\left(\Delta E_{d}, p_{\vec{V}_{j}(t)}\right), \quad j \in\left(1, N_{v i b}\right)
$$

As demonstrated in our previous study, ${ }^{11}$ the production of correlated TT pair from photoexcited $S_{1} S_{0}$ state is primarily through the direct mechanism without the aid of any charge-transfer intermediate. Therefore, $\Delta E_{d}=E_{S_{1} S_{0}}-E_{T T}$, where the $S_{1} S_{0}$ reactant state is modeled by the linear response time-dependent density functional theory (TDDFT), ${ }^{75}$ while the multiconfigurational TT product state $^{76}$ is constructed by the constrained density functional theory ${ }^{77}$ that breaks spin symmetry on reference orbitals through spin-polarized and position-dependent Hartree potential. As shown in Fig. S4(B), our calculated light absorption spectrum is well in line with the experimental UV-Vis spectrum [Fig. S4(A)] by exhibiting two most prominent peaks at $848 \mathrm{~nm}$ and $712 \mathrm{~nm}$. As a result, an incident wavelength of $801 \mathrm{~nm}$ was deliberately chosen in our transient infrared spectroscopy experiment to excite hexacene to its $S_{1} S_{0}$ state rather than its higher-lying electronic states.

In the present study, 5000 snapshots were randomly selected from a 5-ns molecular dynamics trajectory for our functional mode analysis. Since a correlated TT pair can be formed along either axis $a$ or axis $b$, we have to consider both $\Delta E_{d}^{a a}=E_{S_{1} S_{0}}-E_{(T T)_{a a}}$ and $\Delta E_{d}^{b b}=E_{S_{1} S_{0}}-E_{(T T)_{b b}}$ when projecting $\Delta E_{d}$ onto the normal modes, $\vec{V}_{g}$, at the ground electronic state. Note that under the independent mode displaced harmonic oscillator (IMDHO) model, ${ }^{78}$ the potential energy surfaces of the ground and $\mathrm{S}_{1} \mathrm{~S}_{0}$ electronic states are simply displaced from one another along any given normal mode, thus preserving its components and its angular frequency. As shown in Fig. 6, the direct $S_{1} S_{0} \rightarrow T T$ transition is predominantly driven by a few normal modes with $\omega \approx 1600 \mathrm{~cm}^{-1}$ regardless of the directionality of the resultant TT state. These critical normal modes turn out to be ring-stretching vibrations collectively exerted by all hexacene molecules in the reaction center (Fig. S6), suggesting the importance of cooperative vibrations particularly in hexacene. 


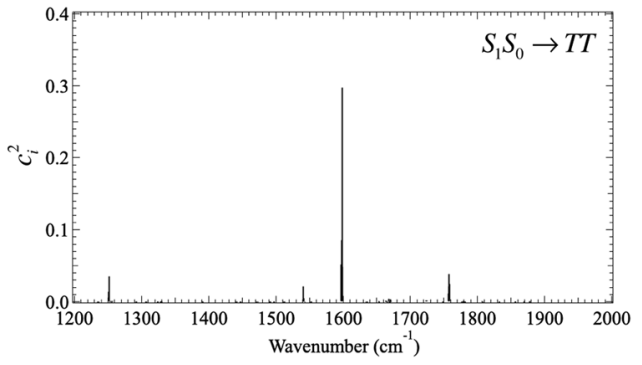

FIG. 6. Calculated spatially averaged reaction coordinates, $\vec{V}_{R C}=\sum c_{i}^{2} \vec{V}_{i}^{\text {ground }}$, for singlet fission, where $\vec{V}_{i}^{\text {ground }}$ denotes the $i$ th normal mode on the ground electronic potential energy surface.

Aiming to reveal the evolution of these critical vibrational modes upon SF, we also projected $\Delta E_{d}$ onto the normal modes of $(T T)_{a a}$ and $(T T)_{b b}$ using the abovementioned functional mode analysis approach. ${ }^{79}$ As shown in Fig. S5, the primary peaks at $\sim 1600 \mathrm{~cm}^{-1}$ of the profiles of $c_{i}^{2}$ underwent a notable split, resulting in a blue shift by $\sim 15 \mathrm{~cm}^{-1}$. If any of those corresponding normal modes exhibits significant Franck-Condon shift upon $S_{0} S_{0} \rightarrow S_{1} S_{0}$ photo-excitation, a blue shift of its transient infrared signal is also expected due to the subsequent $S_{1} S_{0} \rightarrow T T$ transition.

Similar to the formula of calculated static infrared intensity [Eq. (1)], its transient counterpart, $T$, arising from the FranckCondon shift upon photo-excitation is given by

$$
T_{i} \propto\left|\frac{\partial E_{\text {optical }}}{\partial Q_{i}}\right|^{2},
$$

where $E_{\text {optical }}$ is the optical gap associated with the $S_{0} S_{0} \rightarrow S_{1} S_{0}$ transition. As shown in Fig. 3 , three groups of normal modes manifest substantial Franck-Condon shifts. They are the ring-twisting modes at $\sim 1250 \mathrm{~cm}^{-1}$ and $\sim 1380 \mathrm{~cm}^{-1}$, the ring-stretching modes at $\sim 1600 \mathrm{~cm}^{-1}$, and the ring-scissoring modes at $\sim 1770 \mathrm{~cm}^{-1}$. Among these three Franck-Condon active groups, only the one at $\sim 1600 \mathrm{~cm}^{-1}$ is also critical to the vibronic-coupling that drives $\mathrm{SF}$ as illustrated in Fig. 6. In this spirit, the time-dependent transient infrared signal centered at $\sim 1600 \mathrm{~cm}^{-1}$ is expected to undergo a significant wavenumber shift, whereas a much smaller shift is anticipated at $\sim 1250 \mathrm{~cm}^{-1}, \sim 1380 \mathrm{~cm}^{-1}$, and $\sim 1770 \mathrm{~cm}^{-1}$. A previous study of hexacene attributed its slower SF, when compared to pentacene, to multiphonon relaxation as suggested by the Redfield theory. ${ }^{8}$ The effective phonon frequency estimated in that work ranges from $800 \mathrm{~cm}^{-1}$ to $1450 \mathrm{~cm}^{-1}$. Our experimental results pinpoint particular IR vibrational modes for SF that are different than the effective phonon in the multiphonon model.

Taken together, a vibronic coupling mechanism in hexacene for SF is sketched in Scheme 2. Upon photoexcitation, hexacene experiences a direct $\mathrm{SF}$ from a photoexcited $\mathrm{S}_{1} \mathrm{~S}_{0}$ state to a correlated triplet TT pair with a nonadiabatic coupling. This nonadiabatic coupling is primarily driven by a ring-stretching vibrational mode of $1620 \mathrm{~cm}^{-1}$, which exhibits a substantial Franck-Condon shift of

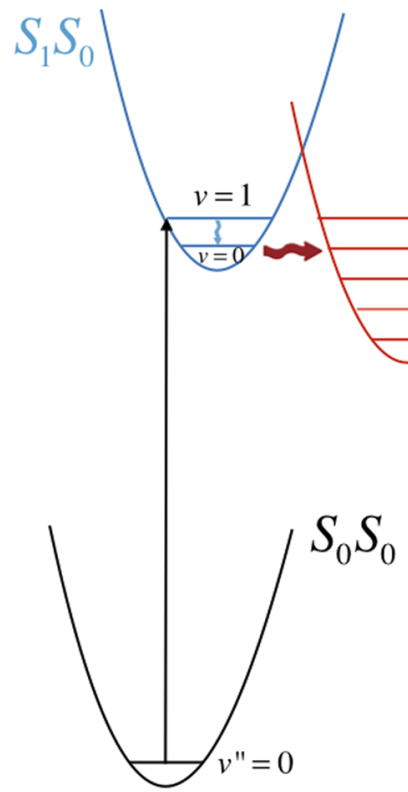

SCHEME 2. Vibronic coupling of $S_{1} S_{0}(v=1)$ and $T T\left(v^{\prime}=3\right)$ in hexacene. The reaction coordinate of this nonadiabatic process is designated as a ring-stretching mode at $\sim 1600 \mathrm{~cm}^{-1}$

$4.0 \mathrm{~cm}^{-1}$. Specifically, this critical vibrational mode is first excited from $S_{0}$ to $S_{1}$ to reach a vibrational quantum number of $v=1$ under the photoillumination of an $801 \mathrm{~nm}$ pump. After an excited-state thermal relaxation, the subsequent $\mathrm{S}_{1} \mathrm{~S}_{0} \rightarrow$ TT transition results in a vibrational heating of $\mathrm{v}^{\prime}=3$ if one considers $\Delta E\left(S_{1} S_{0} \rightarrow T T\right)$ $\approx 0.6 \mathrm{eV}$ and $\omega_{\text {vib }} \approx 1600 \mathrm{~cm}^{-1}$. In fact, vibronic coupling is not only important in exothermic SF systems such as hexacene but also is vital for endothermic ones. For example, a recent study by Friend and co-worker reported vibronically coherent ultrafast triplet-pair formation mediated by the strong coupling of electronic and vibrational degrees of freedom in TIPS-tetracene films. ${ }^{21}$ Another study of TIPS-tetracene nanoparticles by Rao and co-workers further discovered different intermediate triplet excitons in endothermic SF if the excitation wavelength is varied, suggesting distinct vibrational relaxation pathways. ${ }^{8}$

\section{SUMMARY AND CONCLUSIONS}

In this work, we have utilized both transient IR spectroscopy and FMSF theory to probe the vibrational dynamics of SF in hexacene. Our transient IR experiments unveiled the formation of vibrationally excited excitons that consist of three groups of normal modes upon photoexcitation. They include ring-twisting modes near $1300.0 \mathrm{~cm}^{-1}$, ring-stretching modes near $1600.0 \mathrm{~cm}^{-1}$, and ring-scissoring modes near $1700.0 \mathrm{~cm}^{-1}$. All of them were well justified by our calculated transient IR intensity using TDDFT. The most striking feature in our time-resolved transient IR spectra is the significant blue-shift of $4.0 \mathrm{~cm}^{-1}$ for a ring-stretching mode near $1600.0 \mathrm{~cm}^{-1}$. Since the relaxation of this mode occurs on a time constant of $0.59 \pm 0.07$ ps that agrees with the SF time scale, 
we designated it as the primary driving mode for SF. Our conclusion gains immediate support from FMSF theory, which confirmed the predominance of the same mode over all others on vibronic coupling between $S_{1} S_{0}$ and TT. Interestingly, this $\sim 1600.0 \mathrm{~cm}^{-1}$ mode is also Franck-Condon active upon the $S_{0} S_{0} \rightarrow S_{1} S_{0}$ transition, making it possibly detectable by other vibrationally resolved transient spectroscopies including transient Raman. To this end, transient IR can be employed together with transient Raman to render a more complete picture of vibronic coupling in SF.

\section{SUPPLEMENTARY MATERIAL}

See the supplementary material for transient IR absorption spectra of a hexacene thin film and its UV-visible spectra; calculated reaction coordinates of $\mathrm{S}_{1} \mathrm{~S}_{0} \rightarrow$ TT transition; and vibrational normal modes with a notable Franck-Condon shift.

\section{ACKNOWLEDGMENTS}

Y. Rao acknowledges support from Utah State University. $\mathrm{H}$. Chen is grateful for the computational resources provided under Department of Energy Contract (No. DE-AC02-06CH11357) and National Science Foundation Contract No. TG-CHE130008. H. Bian acknowledges the support from the Natural Science Foundation of China (NSFC, Grant Nos. 21603137 and 21873062) and Fundamental Research Funds for the Central Universities (GK201701004). This work was also supported by the Key Laboratory of Applied Surface and Colloid Chemistry (Shaanxi Normal University).

The authors declare no competing financial interests.

\section{REFERENCES}

${ }^{1}$ R. E. Merrifield, P. Avakian, and R. P. Groff, Chem. Phys. Lett. 3, 155 (1969).

${ }^{2}$ M. B. Smith and J. Michl, Chem. Rev. 110, 6891 (2010).

${ }^{3}$ A. Rao and R. H. Friend, Nat. Rev. Mater. 2, 17063 (2017).

${ }^{4}$ M. W. B. Wilson, A. Rao, J. Clark, R. S. S. Kumar, D. Brida, G. Cerullo, and R. H. Friend, J. Am. Chem. Soc. 133, 11830 (2011).

${ }^{\mathbf{5}}$ B. J. Walker, A. J. Musser, D. Beljonne, and R. H. Friend, Nat. Chem. 5, 1019 (2013).

${ }^{6}$ W. L. Chan, M. Ligges, A. Jailaubekov, L. Kaake, L. Miaja-Avila, and X. Y. Zhu, Science 334, 1541 (2011).

${ }^{7}$ W. L. Chan, M. Ligges, and X. Y. Zhu, Nat. Chem. 4, 840 (2012).

${ }^{8}$ E. Busby, T. C. Berkelbach, B. Kumar, A. Chernikov, Y. Zhong, H. Hlaing, X. Y. Zhu, T. F. Heinz, M. S. Hybertsen, M. Y. Sfeir, D. R. Reichman, C. Nuckolls, and O. Yaffe, J. Am. Chem. Soc. 136, 10654 (2014).

${ }^{9}$ A. A. Bakulin, S. E. Morgan, T. B. Kehoe, M. W. B. Wilson, A. W. Chin, D. Zigmantas, D. Egorova, and A. Rao, Nat. Chem. 8, 16 (2016).

${ }^{10}$ S. W. Eaton, L. E. Shoer, S. D. Karlen, S. M. Dyar, E. A. Margulies, B. S. Veldkamp, C. Ramanan, D. A. Hartzler, S. Savikhin, T. J. Marks, and M. R. Wasielewski, J. Am. Chem. Soc. 135, 14701 (2013).

${ }^{11}$ N. R. Monahan, D. Sun, H. Tamura, K. W. Williams, B. Xu, Y. Zhong, B. Kumar, C. Nuckolls, A. R. Harutyunyan, G. Chen, H.-L. Dai, D. Beljonne, Y. Rao, and X. Y. Zhu, Nat. Chem. 9, 341 (2017).

${ }^{12}$ J. J. Burdett and C. J. Bardeen, J. Am. Chem. Soc. 134, 8597 (2012).

${ }^{13}$ A. K. Le, J. A. Bender, D. H. Arias, D. E. Cotton, J. C. Johnson, and S. T. Roberts, J. Am. Chem. Soc. 140, 814 (2018).

${ }^{14}$ N. A. Pace, W. Zhang, D. H. Arias, I. McCulloch, G. Rumbles, and J. C. Johnson, J. Phys. Chem. Lett. 8, 6086 (2017).

${ }^{15}$ J. Hu, K. Xu, L. Shen, Q. Wu, G. He, J.-Y. Wang, J. Pei, J. Xia, and M. Y. Sfeir, Nat. Commun. 9, 2999 (2018)
${ }^{16}$ B. Zhang, C. Zhang, Y. Xu, R. Wang, B. He, Y. Liu, S. Zhang, X. Wang, and M. Xiao, J. Chem. Phys. 141, 244303 (2014).

${ }^{17}$ C. K. Yong, A. J. Musser, S. L. Bayliss, S. Lukman, H. Tamura, O. Bubnova, R. K. Hallani, A. Meneau, R. Resel, M. Maruyama, S. Hotta, L. M. Herz, D. Beljonne, J. E. Anthony, J. Clark, and H. Sirringhaus, Nat. Commun. 8, 15953 (2017).

${ }^{18}$ R. D. Pensack, E. E. Ostroumov, A. J. Tilley, S. Mazza, C. Grieco, K. J. Thorley, J. B. Asbury, D. S. Seferos, J. E. Anthony, and G. D. Scholes, J. Phys. Chem. Lett. 7, 2370 (2016).

${ }^{19}$ K. Miyata, Y. Kurashige, K. Watanabe, T. Sugimoto, S. Takahashi, S. Tanaka, J. Takeya, T. Yanai, and Y. Matsumoto, Nat. Chem. 9, 983 (2017).

${ }^{20}$ A. J. Musser, M. Liebel, C. Schnedermann, T. Wende, T. B. Kehoe, A. Rao, and P. Kukura, Nat. Phys. 11, 352 (2015).

${ }^{21}$ H. L. Stern, A. Cheminal, S. R. Yost, K. Broch, S. L. Bayliss, K. Chen, M. Tabachnyk, K. Thorley, N. Greenham, J. M. Hodgkiss, J. Anthony, M. HeadGordon, A. J. Musser, A. Rao, and R. H. Friend, Nat. Chem. 9, 1205 (2017).

${ }^{22}$ Y. Wan, Z. Guo, T. Zhu, S. Yan, J. Johnson, and L. Huang, Nat. Chem. 7, 785 (2015).

${ }^{23}$ B. D. Folie, J. B. Haber, S. Refaely-Abramson, J. B. Neaton, and N. S. Ginsberg, J. Am. Chem. Soc. 140, 2326 (2018).

${ }^{24}$ S. Lukman, K. Chen, J. M. Hodgkiss, D. H. P. Turban, N. D. M. Hine, S. Dong, J. Wu, N. C. Greenham, and A. J. Musser, Nat. Commun. 7, 13622 (2016).

${ }^{25}$ A. J. Musser, M. Al-Hashimi, M. Maiuri, D. Brida, M. Heeney, G. Cerullo, R. H. Friend, and J. Clark, J. Am. Chem. Soc. 135, 12747 (2013).

${ }^{26}$ P. M. Zimmerman, Z. Y. Zhang, and C. B. Musgrave, Nat. Chem. 2, 648 (2010).

${ }^{27}$ P. M. Zimmerman, F. Bell, D. Casanova, and M. Head-Gordon, J. Am. Chem. Soc. 133, 19944 (2011).

${ }^{28}$ T. C. Berkelbach, M. S. Hybertsen, and D. R. Reichman, J. Chem. Phys. 138, 114103 (2013).

${ }^{29}$ R. Tempelaar and D. R. Reichman, J. Chem. Phys. 146, 174703 (2017).

${ }^{30}$ H. Tamura, M. Huix-Rotllant, I. Burghardt, Y. Olivier, and D. Beljonne, Phys. Rev. Lett. 115, 107401 (2015).

${ }^{31}$ D. Casanova, Chem. Rev. 118, 7164 (2018).

${ }^{32}$ A. F. Morrison and J. M. Herbert, J. Phys. Chem. Lett. 8, 1442 (2017).

${ }^{33}$ M. Andrzejak, T. Skóra, and P. Petelenz, J. Phys. Chem. C 123, 91 (2019).

${ }^{34}$ A. Japahuge and T. Zeng, ChemPlusChem 83, 146 (2018).

${ }^{35}$ L. A. Martínez-Martínez, M. Du, R. F. Ribeiro, S. Kéna-Cohen, and J. Yuen-Zhou, J. Phys. Chem. Lett. 9, 1951 (2018).

${ }^{36}$ Y. Fujihashi, L. Chen, A. Ishizaki, J. Wang, and Y. Zhao, J. Chem. Phys. 146, 044101 (2017).

${ }^{37}$ H. Zang, Y. Zhao, and W. Liang, J. Phys. Chem. Lett. 8, 5105 (2017).

${ }^{38}$ S. R. Yost, J. Lee, M. W. B. Wilson, T. Wu, D. P. McMahon, R. R. Parkhurst, N. J. Thompson, D. N. Congreve, A. Rao, K. Johnson, M. Y. Sfeir, M. G. Bawendi, T. M. Swager, R. H. Friend, M. A. Baldo, and T. Van Voorhis, Nat. Chem. 6, 492 (2014).

${ }^{39}$ N. Renaud and F. C. Grozema, J. Phys. Chem. Lett. 6, 360 (2015).

${ }^{40}$ S. Ito, T. Nagami, and M. Nakano, J. Phys. Chem. Lett. 6, 4972 (2015).

${ }^{41}$ E. C. Alguire, J. E. Subotnik, and N. H. Damrauer, J. Phys. Chem. C 119, 299 (2015).

${ }^{42}$ T. Zeng, R. Hoffmann, and N. Ananth, J. Am. Chem. Soc. 136, 5755 (2014).

${ }^{43}$ E. A. Buchanan and J. Michl, J. Am. Chem. Soc. 139, 15572 (2017).

${ }^{44}$ E. C. Greyson, J. Vura-Weis, J. Michl, and M. A. Ratner, J. Phys. Chem. C 114, 14168 (2010).

${ }^{45}$ J. Wen, Z. Havlas, and J. Michl, J. Am. Chem. Soc. 137, 165 (2015).

${ }^{46}$ J. E. Elenewski, U. S. Cubeta, E. Ko, and H. Chen, J. Phys. Chem. C 121, 11159 (2017).

${ }^{47}$ R. A. Marcus, Annu. Rev. Phys. Chem. 15, 155 (1964).

${ }^{48}$ C. Grieco, E. R. Kennehan, H. Kim, R. D. Pensack, A. N. Brigeman, A. Rimshaw, M. M. Payne, J. E. Anthony, N. C. Giebink, G. D. Scholes, and J. B. Asbury, J. Phys. Chem. C 122, 2012 (2018).

${ }^{49}$ C. Grieco, E. R. Kennehan, A. Rimshaw, M. M. Payne, J. E. Anthony, and J. B. Asbury, J. Phys. Chem. Lett. 8, 5700 (2017). 
${ }^{50}$ M. Chen, Y. J. Bae, C. M. Mauck, A. Mandal, R. M. Young, and M. R. Wasielewski, J. Am. Chem. Soc. 140, 9184 (2018).

${ }^{51}$ E. A. Margulies, N. Kerisit, P. Gawel, C. M. Mauck, L. Ma, C. E. Miller, R. M. Young, N. Trapp, Y.-L. Wu, F. Diederich, and M. R. Wasielewski, J. Phys. Chem. C 121, 21262 (2017)

${ }^{52}$ Q. Wei, D. Zhou, X. Li, Y. Chen, and H. Bian, J. Phys. Chem. B 122, 12131 (2018).

${ }^{53}$ D. Zhou, Q. Wei, S. Wang, X. Li, and H. Bian, J. Phys. Chem. Lett. 10, 176 (2019).

${ }^{54}$ M. Watanabe, Y. J. Chang, S.-W. Liu, T.-H. Chao, K. Goto, M. M. Islam, C.-H. Yuan, Y.-T. Tao, T. Shinmyozu, and T. J. Chow, Nat. Chem. 4, 574 (2012).

${ }^{55}$ A. Warshel and M. Levitt, J. Mol. Biol. 103, 227 (1976).

${ }^{56}$ S. Goedecker, M. Teter, and J. Hutter, Phys. Rev. B 54, 1703 (1996).

${ }^{57}$ D. Jacquemin, B. Moore, A. Planchat, C. Adamo, and J. Autschbach, J. Chem. Theory Comput. 10, 1677 (2014).

${ }^{58}$ D. E. Woon and T. H. Dunning, J. Chem. Phys. 100, 2975 (1994).

${ }^{59}$ T. Laino, F. Mohamed, A. Laio, and M. Parrinello, J. Chem. Theory Comput. 1, 1176 (2005).

${ }^{60}$ L. Genovese, T. Deutsch, A. Neelov, S. Goedecker, and G. Beylkin, J. Chem. Phys. 125, 074105 (2006).

${ }^{61}$ J. Wang, R. M. Wolf, J. W. Caldwell, P. A. Kollman, and D. A. Case, J. Comput. Chem. 25, 1157 (2004).

${ }^{62}$ J. VandeVondele, M. Krack, F. Mohamed, M. Parrinello, T. Chassaing, and J. Hutter, Comput. Phys. Commun. 167, 103 (2005).

${ }^{63}$ C. L. Darling and H. B. Schlegel, J. Phys. Chem. 98, 5855 (1994).

${ }^{64} \mathrm{M}$. Bass, E. Van Stryland, D. Williams, and W. Wolfe, Handbook of Optics Volume II: Devices, Measurements and Properties (OSA, 2000), Vol. 5.
${ }^{65}$ K. Lee, R. A. J. Janssen, N. S. Sariciftci, and A. J. Heeger, Phys. Rev. B 49, 5781 (1994).

${ }^{66}$ N. A. Anderson, E. Hao, X. Ai, G. Hastings, and T. Lian, Chem. Phys. Lett. 347, 304 (2001).

${ }^{67}$ M. Tong, C. X. Sheng, C. Yang, Z. V. Vardeny, and Y. Pang, Phys. Rev. B 69, 155211 (2004).

${ }^{68}$ A. S. Dhoot, D. S. Ginger, D. Beljonne, Z. Shuai, and N. C. Greenham, Chem. Phys. Lett. 360, 195 (2002).

${ }^{69}$ R. D. Pensack and J. B. Asbury, J. Am. Chem. Soc. 131, 15986 (2009).

${ }^{70}$ R. D. Pensack, K. M. Banyas, L. W. Barbour, M. Hegadorn, and J. B. Asbury, Phys. Chem. Chem. Phys. 11, 2575 (2009).

${ }^{71}$ J. Guo, H. Ohkita, H. Benten, and S. Ito, J. Am. Chem. Soc. 131, 16869 (2009).

${ }^{72}$ A. De Sio, F. Troiani, M. Maiuri, J. Réhault, E. Sommer, J. Lim, S. F. Huelga, M. B. Plenio, C. A. Rozzi, G. Cerullo, E. Molinari, and C. Lienau, Nat. Commun. 7, 13742 (2016).

${ }^{73}$ P. N. Prasad, Mol. Cryst. Liq. Cryst. 58, 39 (1980).

${ }^{74}$ H. Chen, J. Phys. Chem. C 118, 7586 (2014).

${ }^{75}$ M. E. Casida and M. Huix-Rotllant, Annu. Rev. Phys. Chem. 63, 287 (2012).

${ }^{76}$ J. E. Elenewski, U. S. Cubeta, E. Ko, and H. Chen, J. Phys. Chem. C 121, 4130 (2017).

${ }^{77}$ B. Kaduk, T. Kowalczyk, and T. Van Voorhis, Chem. Rev. 112, 321 (2012).

${ }^{78}$ J. R. Reimers, K. R. Wilson, and E. J. Heller, J. Chem. Phys. 79, 4749 (1983).

${ }^{79}$ J. S. Hub and B. L. de Groot, PLOS Comput. Biol. 5, e1000480 (2009).

${ }^{80}$ A. Petrone, D. B. Lingerfelt, D. B. Williams-Young, and X. Li, J. Phys. Chem. Lett. 7, 4501 (2016).

${ }^{81}$ A. Thampi, H. L. Stern, A. Cheminal, M. J. Y. Tayebjee, A. J. Petty, J. E. Anthony, and A. Rao, J. Am. Chem. Soc. 140, 4613 (2018). 